\title{
An explanation for the presentation-order effect in the method of constant stimuli
}

\author{
SERGIO CESARE MASIN \\ University of Udine, Udine, Italy \\ and \\ VALENTINA FANTON \\ University of Padua, Padua, Italy
}

\begin{abstract}
The point of subjective equality obtained by the method of constant stimuli depends to a great extent on whether the standard (S) or the variable (V) stimulus occurs first. This presentationorder effect was studied using lines as stimuli. Successive $S, V$ pairs were presented, with interstimulus and interpair intervals equal. Observers, who were not told which was $S$ or $V$, reported whether a given line was longer or shorter than the immediately preceding line. Although the observers' subjective experience was of a train of lines that was not organized into pairs, the presentation-order effect still occurred. This inplies that the effect does not depend on the order of presentation of the stimuli in an experienced pair. It was also shown that the observers could categorize line lengths, since they could identify stochastically the most frequent stimulus (S). We propose that the presentation-order effect depends on a decision process based on response probabilities inferred from length categories.
\end{abstract}

In the method of constant stimuli (MCS) for the determination of the point of subjective equality (PSE), the standard (S) stimulus is paired with a variable (V) stimulus. The observer's task is to report whether one of the two stimuli is greater or less than the other with respect to a given attribute. The $S$, usually physically equal to one of the Vs, is chosen so that the probability of detecting a difference between the $S$ and $a \mathrm{~V}$ is greater than 0 and less than 1 .

The version of the MCS in which the $S$ and the $V$ in a pair are presented successively is known to produce a time error whose magnitude depends on a variety of factors (Guilford, 1954; Hellström, 1985; Woodworth \& Schlosberg, 1954). One such factor is the presentation order of $S$ and $V$ in a pair (Fechner, 1860; Masin, 1987; Woodruff, Jennings, \& Rico, 1975; see Needham, 1934, for a review of early studies). The PSE tends to be displaced away from (toward) the center of the range of Vs when $S$ appears second (first) in a pair. The effect occurs for different kinds of stimuli, including lines (Masin, 1988).

However, the presentation-order effect may be independent of the order of presentation in an experienced pair. As far as we know, in all studies on the MCS, the interstimulus interval was different from the interpair interval. This procedure assumes that the difference between the two intervals has no effect on the PSE. If this were true, then it should also be true that the order of presen-

This research was supported by a grant from the Italian Research Council (CNR). We thank M. Teghtsoonian for helpful comments on the manuscript. Correspondence may be addressed to S. C. Masin, Institute of Philosophy, Pedagogics, and Teaching of Modern Languages, Via Antonini 8, 33100 Udine, Italy. tation in an experienced pair has no effect on the PSE, because in this respect, two sequences-one of pairs $S, V$ and one of pairs V,S-are indistinguishable.

In Experiment 1, we tested whether order of presentation in an experienced pair has an effect on the PSE by making the interstimulus and interpair intervals equal. The result was a train of stimuli that produced no experience of pairs and, thus, to which the concept of a perceived presentation order of $\mathbf{S}$ and $\mathbf{V}$ is not applicable.

\section{EXPERIMENT 1}

\section{Method}

Observers. The observers were 80 university students who were asked to participate in the experiment as they entered the General Psychology Department at the University of Padua. Their declared vision was normal or corrected by lenses.

Stimuli. The stimuli, red or green horizontal lines of 1.5-sec duration, were displayed singly in the center of a $17.5 \times 23.5 \mathrm{~cm}$ screen of a Taxan monitor controlled by an Apple microcomputer. The center of each line coincided with the center of the screen. The lines appeared moderately bright on a nonluminous dark gray background. Red and green lines appeared equally bright. The illumination level was $41 x$.

As in the usual MCS procedure, $S$ and $V$ were presented in pairs. Both the interstimulus interval (between $S$ and the succeeding $V$ ) and the interpair interval (between $\mathrm{V}$ and the succeeding $\mathrm{S}$ ) were $5 \mathrm{sec}$. This resulted in a train of lines separated from one another by the same time interval, with no temporal grouping into pairs. The length of V (V1, V2, ., or V5) was, respectively, 99.2, $102.5,105.8,109.1$, and $112.4 \mathrm{~mm}$ on the screen. The length of S (=V4) was $109.1 \mathrm{~mm}$. Each stimulus was announced by a very brief acoustic signal $1.5 \mathrm{sec}$ before its appearance.

The observer sat at a small table provided with a chinrest to beep the head level with the stimuli. To avoid reflections of objects on the monitor screen, a black (reflectance .05 ) $130 \times 130 \mathrm{~cm}$ rect- 
angular screen was interposed between the observer's head and the monitor. The black screen had an $8 \times 13 \mathrm{~cm}$ rectangular hole through which the observer viewed the monitor binocularly. The black screen was $25 \mathrm{~cm}$ from the observer's face, and the monitor screen was $95 \mathrm{~cm}$ from the black screen.

Procedure. (For half of the observers, all the lines were red, and for the other half, green. This color difference is irrelevant to the present research.) The observer's task was to report whether each line on the monitor screen was longer or shorter than the immediately preceding line. The responses "equal" and "don't know" were not allowed. Ten consecutive series of pairs $S, V$ were presented; a series is defined as a sequence of five pairs of $S$ coupled with each one of V1-V5, which were presented in a different random order for each observer. The observers started responding after the second line appeared. To compensate for the absence of a response to the first line (an S), a final $S$ was presented at the end of the 10 series. Thus, each observer gave 100 responses in total, 50 to the $S$ and 50 to the Vs. The observers were not told which was $\mathrm{S}$ or $\mathrm{V}$. They were asked to respond as soon as possible within 5 sec after a line appeared.

\section{Results and Discussion}

It is an explicit "longer" response to $\mathrm{V}$ when the observer reports "longer" to $\mathrm{V}$ following $\mathrm{S}$, and an implicit "longer" response when he/she reports "shorter" to S following V. The mean PSEs from these explicit and implicit "longer" responses were computed by fitting the psychometric functions by the weighted least squares procedure. The mean PSEs were computed separately for the responses given in each series.

The overall results for the entire group of 80 observers are represented in Figure 1 and clearly show that the presentation-order effect still occurred when the stimuli were members of a train of lines in which no pairs were defined by temporal grouping. (The two groups of $40 \mathrm{ob}-$ servers who responded only to red or green lines showed the same pattern of results.) The PSE was roughly one

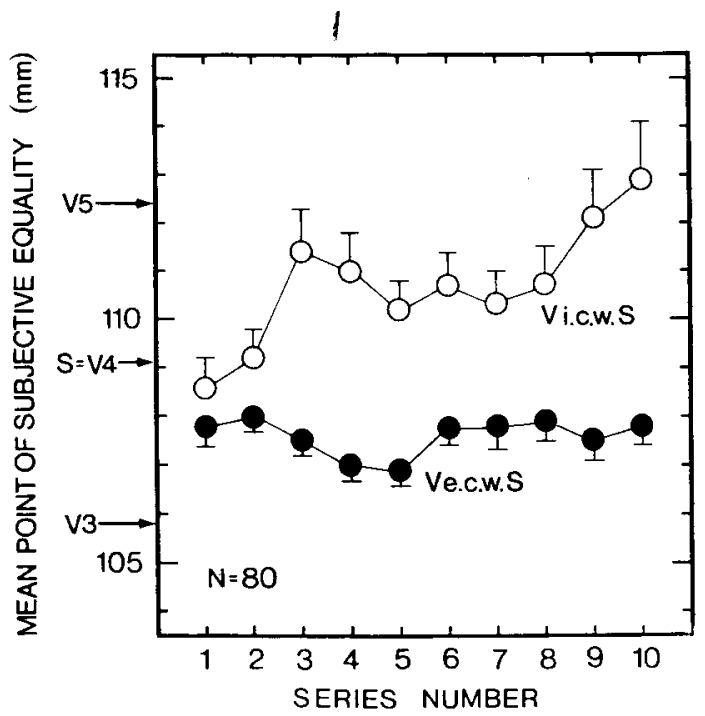

Figure 1. Results of Experiment 1. Mean point of subjective equality (PSE) from the method of constant stimuli as a function of series number. The filled and empty circles represent the mean PSEs from the explicit (V e.c.w. S) and implicit (V i.c.w. S) comparisons of $V$ with $S$, respectively. third of a centimeter greater (about $3 \%$ of $S$ ) when the response was made to $S$ (following $V$ ) than when it was made to $\mathrm{V}$ (following $\mathrm{S}$ ). (Compare these results with those of Masin, 1987, and Masin, Mazzoni, \& Vallortigara, 1987.)

If we assume that no pairs of lines were experienced, it must be concluded that the presentation-order effect did not depend on the order of presentation of stimuli within an experienced pair. Therefore, it may also be conlcuded that the implicit assumption, made in all previous studies-that the difference in duration of the interstimulus and interpair intervals was not influential-is confirmed.

\section{EXPERIMENT 2}

This experiment served to check whether the results of Experiment 1 were due to a response preference. If the mode of judgment is changed so that the stimuli in Experiment 1 that received "longer" responses now receive "shorter" responses, the presentation-order effect should be reversed if a preference for the "longer" response caused it.

\section{Method}

Observers. The observers were 45 different university students who were recruited as in Experiment 1.

Stimuli. The apparatus was the same as in Experiment 1. The stimuli were also the same, except that the lines were orange and the lengths of V1-V5 were 87.2, 89.9, 92.6, 95.3 , and $98.0 \mathrm{~mm}$, respectively. The standard was V4.

Procedure. The procedure was the same as in Experiment 1 except that now the observers were asked to report whether each line that immediately preceded, rather than followed, the line currently on the monitor screen was longer or shorter than this line on the monitor screen. (Methodologically, it is important to note that observers have difficulty performing the task because there is a strong tendency to execute the opposite task; that is, to compare the line on the monitor screen with the preceding line. Thus, the observers had to be urged to be extremely careful and attentive.)

\section{Results}

It is an explicit "longer" response to $\mathrm{V}$ when the observer reports "longer" to $\mathrm{V}$ preceding $\mathrm{S}$, and an implicit "longer" response when he/she reports "shorter" to $S$ preceding V. The constant error (PSE-S) from the implicit "longer" response to V compared with the previous $S$ was -0.8 , and the constant error from the explicit "longer" response to $\mathrm{V}$ compared with the following $\mathrm{S}$ was 1.49. Both constant errors were significant at the .001 level, $z \mathrm{~s}=4.7$ and 4.3 , respectively, assuming statistical independence between series. Since the constant errors had the same sign as in Experiment 1, it may be concluded that the presentation-order effect was not affected substantially by a response preference.

\section{EXPERIMENT 3}

Masin et al. (1987) have shown that the presentationorder effect occurs from the second response of the ob- 
server. The only sensory information immediately available for the production of a response is the absolute magnitudes of $\mathrm{S}$ or $\mathrm{V}$. It seems reasonable that observers immediately encode, or categorize, this information, and later use the codes or categories for the comparison judgment (Bower, 1971).

In Experiment 3, we tested the hypothesis that observers are able to categorize the magnitudes of $S$ and Vs (e.g., into short, long, and medium line lengths). Observers were asked to detect the higher frequency of occurrence of $S$ in the train of lines used in Experiment 1. Since they were not told which was $S$ or $V$, identification of $S$ was possible only if categorization occurred.

\section{Method}

Observers. The observers were 30 different university students who were recruited as in Experiments 1 and 2.

Stimuli. The apparatus and the stimuli were the same as in Experiment 1 , except that the lines were green for all observers.

Procedure. Each observer was told that a train of horizontal lines was going to be shown, and that one of those lines would appear many times. He/she was to compare each line with the immediately preceding one, but to report only whether the line seen was the line that occurred most frequently, as scon as possible within $5 \mathrm{sec}$. No other information about the $S$ or the Vs was given to the observer. The random order of presentation of Vs was different for each observer.

\section{Results and Discussion}

The results are reported in Figure 2. Since $S=V 4$, in each series there were six physical Ss. The mean number of times that the five Ss plus V4, or V3 plus V5, or V1 plus V2, were reported to be the most frequent line is represented on the ordinate as a function of the series number. Chance responding would produce mean numbers oscillating around 15 (30 observers). As may be seen,

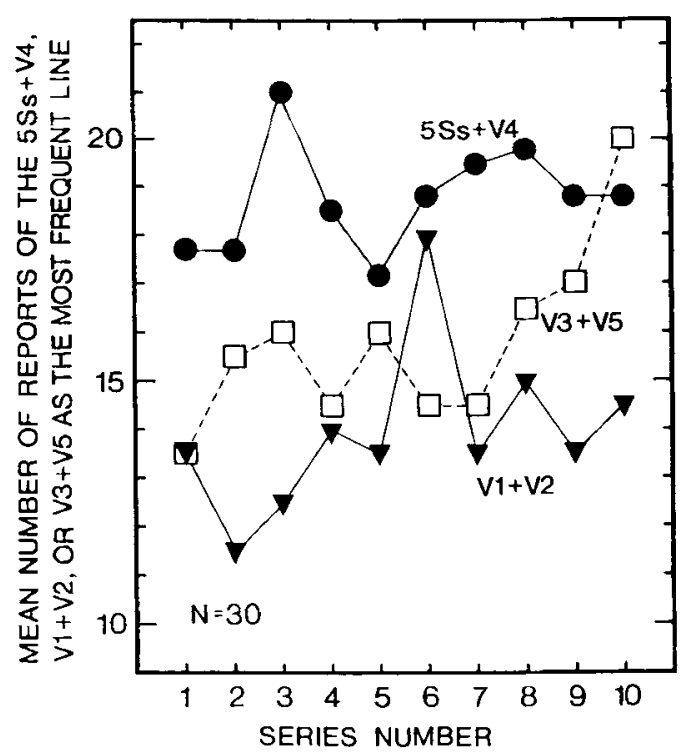

Figure 2. Results of Experiment 3. Mean number of times the five Ss plus V4 (or V1 plus V2, or V3 plus V5) were reported to be the most frequent stimulus, as a function of series number. the mean number of times each $S$ was reported to be the most frequent stimulus is well above 15 in all 10 series. This result confirms that $\mathbf{S}$ was stochastically identified by observers as the most frequent stimulus.

Another result is noteworthy. As Figure 2 evidences, the closer in value a given $V$ was to $S$, the greater was the probability it was mistakenly reported as the most frequent stimulus.

The observer's capacity to detect the higher frequency of a stimulus ( $S$ in our experiment) without the knowledge of which was $\mathrm{S}$ or $\mathrm{V}$ implies that the stimuli were categorized as having a given length, for example as being long, short, or medium in length.

Figure 2 might give the impression that the most frequently presented line (S) was recognized. If that were so, then in Experiment 1, the S,V or V,S pairs could have been distinguished from one another just because $S$ was recognized each time. However, $S$ was not recognized in either Experiment 1 or Experiment 3. In fact, the observers reported no detection of any sort of pair in either experiment. In addition, consider that Figure 2 shows that $S$ was correctly selected as most frequent on only $65 \%$ of the occasions on which it was judged, and that V1 + V2 and V3 + V5 were incorrectly selected as most frequent on $40 \%$ and $50 \%$, respectively, of the occasions on which they were judged. The frequency of errors makes it unlikely that the observers were identifying S,V or $\mathrm{V}, \mathrm{S}$ pairs.

\section{GENERAL DISCUSSION}

These results allow for the following interpretation of the presentation-order effect in the MCS. Experimental data and theoretical reflection suggest that, in the comparative judgment of two stimuli, (1) the stimuli are automatically categorized according to their magnitude, and (2) the difference judgment is derived from a comparison of the categories to which the stimuli are assigned (Bower, 1971; Potts et al., 1978). We further assume that (3) the categorical comparison is accompanied by an additional inferential decision process that uses only the category relative to the second stimulus because more weight is given to this category, or because the category relative to the first stimulus is momentarily forgotten. Thus, if the second stimulus is categorized as long, then it must have a high probability of being longer. Therefore, the "longer" response tends to be produced on the basis of that inferred probability. On the contrary, if the stimulus is categorized as short, then it must have a high probability of being shorter, and this inferred probability is plausibly used to produce the "shorter" response. Figure 3 illustrates time errors in the MCS that may be explained by this interpretation.

Figures $3 a$ and $3 b$ illustrate the psychometric functions for the "longer" response given to the Vs, in the extreme cases when $S=V 1$ and $S=V 5$, respectively. Figures $3 \mathrm{c}$ and $3 \mathrm{~d}$ illustrate the psychometric functions for the "longer" responses given to the $S$ that immediately fol- 

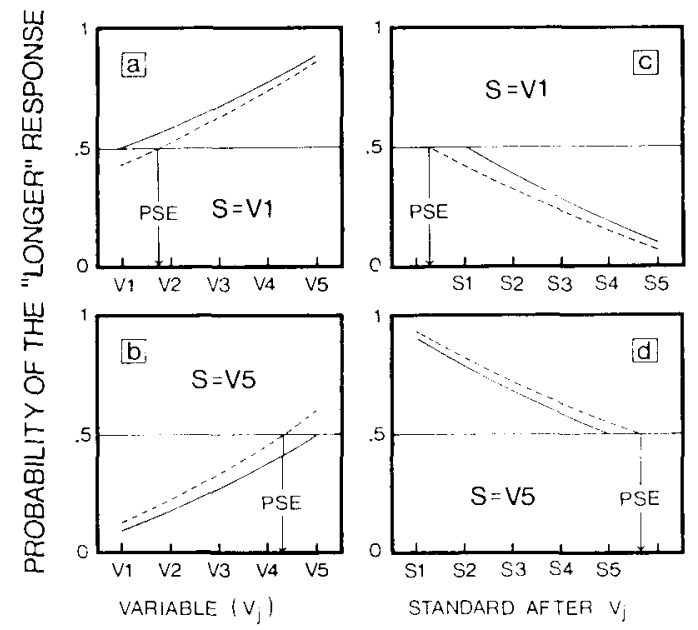

Figure 3. Psychometric functions from the "longer" responses given to Vs and Ss in the extreme cases when $S=V 1$ and $S=V 5$. The solid (dashed) curves represent the theoretical (observed) functions.

lowed a given $\mathrm{V}$, in the extreme cases when $\mathrm{S}=\mathrm{V} 1$ and $S=V 5$, respectively. ( $S 1, S 2, \ldots, S 5$ denote the $S$ immediately following $\mathrm{V} 1, \mathrm{~V} 2, \ldots, \mathrm{V} 5$, respectively, and are represented at equal distances on the same continuum as the Vs.) The solid curves in Figure 3 represent the theoretical psychometric functions in the case in which there is no presentation-order effect and no time error, whereas the dashed curves represent the observed psychometric functions in the case in which there is a presentation-order effect.

In the extreme case when $S=V 1$, the Vs close to $S$ are short lines and, therefore, receive additional "shorter" responses (due to the inferred probability that lines should be shorter if they are categorized as short); $\mathrm{S}$ is also a short line, and therefore it also receives additional "shorter" responses for the same reason. This addition of "shorter" responses implies the obvious decrease in the number of "longer" responses. The dashed psycho- metric functions in Figures $3 \mathrm{a}$ and $3 \mathrm{c}$ would be the result of that decrease.

Similarly, in the extreme case when $S=V 5$, the Vs close to $S$ are long lines, and so receive additional "longer" responses; $\mathrm{S}$ is also a long line, and therefore it also receives additional "longer" responses. The dashed psychometric functions in Figures $3 b$ and $3 d$ would be the result of the increase in the number of "longer" responses produced by the inference that lines that are categorized as long should also have a high probability of being longer.

\section{REFERENCES}

Bower, G. (1971). Adaptation-level coding of stimuli and serial position effects. In M. H. Appley (Ed.), Adaptation-level theory (pp. 175201). New York: Academic Press.

Fechner, G. T. (1860). Elemente der Psychophysik. Leipzig: Breitkopf \& Hartel.

GUILFORD, J. P. (1954). Psychometric methods. New York: McGraw-Hill.

Hellström, A. (1985). The time-order error and its relatives: Mirrors of cognitive processes in comparing. Psychological Bulletin, 97, 35-61.

MAsin, S. C. (1987). Different biases in the methods of constant and single stimuli. Bulletin of the Psychonomic Society, 25, 379-382.

Masin, S. C. (1988, November). New data and an explanation for the time- and space-errors. Paper presented at the 29th Annual Meeting of the Psychonomic Society, Chicago.

Masin, S. C., Mazzoni, G., \& Vallortigara, G. (1987). The first five responses in the method of constant stimuli. Canadian Journal of Psychology, 41, 80-83.

NeEdham, J. G. (1934). The time error in comparison judgments. Psychological Bulletin, 31, 229-243.

Potts, G. R., Banks, W. P., Kosslyn, S. M., Moyer, R. S., Riley, C. A., SMITH, K. H. (1978). Encoding and retrieval in comparative judgment. In N. J. Castellan, Jr. \& F. Restle (Eds.), Cognitive theory (pp. 243-308). Hillsdale, NJ: Erlbaum.

WoOdrufF, B., Jennings, D. L., \& Rico, N. L. (1975). Time error in lifted weights as affected by presentation order and judgment mode. Perception \& Psychophysics, 18, 98-104.

WOODWORTH, R. S., \& SCHLOSBERG, H. (1954). Experimental psychology. New York: Holt.

(Manuscript received May 12, 1987; revision accepted for publication April 26, 1989.) 